\title{
Estimating Visitor Use at Attraction Sites and Trailheads in Yosemite National Park Using Automated Visitor Counters
}

\author{
David Pettebone, Ph.D. \\ Social Scientist \\ Yosemite National Park \\ PO Box 700-W \\ El Portal, CA 95318 \\ Peter Newman, Ph.D. \\ Associate Dean of Academic Affairs \\ Colorado State University \\ 233 Forestry Building \\ Fort Collins, CO 80523 \\ Steve Lawson, Ph.D. \\ Senior Project Consultant \\ Resources Systems Group \\ 55 Railroad Row \\ White River Junction, VT 05001
}




\section{Introduction}

Baseline data on visitor use is essential for informed planning and management of national park units, as such information provides an empirical basis to: 1) assess visitor impacts to the resource; 2) estimate the quality of the visitor experience; and 3) guide visitor facilities planning (Loomis, 2000). Further, in a survey of state and federal land managers in the United States of America (U.S.A.), respondents identified visitor use data as being necessary to inform trail planning projects, justify funding, and support day-to-day trail management (Lynch, Vogt, Cindrity, \& Nelson, 2002). However, visitor use data are not always available and can be difficult to collect. While all national park units are required to track overall visitor use levels (i.e, parkwide visitation), more spatially precise information about the amount of visitor use (e.g., visitation to key venues within a national park unit) is much less commonly recorded because of the costs and challenges of doing so. In fact, lack of funding and personnel time, logistical problems, and lack of training about available methods to collect and analyze visitation data have been identified as primary reasons why visitor use has not been measured more systematically in national parks and related protected natural areas (Watson, Cole, Turner, \& Reynolds, 2000).

Recently, automated visitor counters have gained recognition as a potentially cost efficient and relatively easy way for land managers to estimate recreational visitation. Park managers and researchers have applied visitor use data to a variety of efforts to inform park planning efforts. Applications of visitor use data to park planning and management include quantifying visitor use at trailheads (Lindsey \& Ngyuen, 2004; Pettebone, Newman, Beaton, Gibson, \& Stack, 2008), developing statistical models of visitor use (Chen, Bloomsfield, \& Fu, 2003; Lawson, Newman, Choi, Pettebone, \& Meldrum, 2008; Lindsey, Han, Wilson, \& Yang, 
2006a; Lindsey \& Lindsey, 2004; Lindsey, Wilson, Rubchinskaya, Yang, \& Han, 2006b), and informing computer simulation modeling of visitor use (Lawson, 2006). Information derived from these analytical efforts provides important baseline data from which to understand the timing and location of visitor use throughout a protected area. Knowledge of current use conditions is critical to developing effective visitor management strategies to ensure natural resources and the quality of the visitor experience are protected (Loomis, 2000; Manning, 2008).

As a tool for collecting visitor use data, automated visitor monitors have the advantages of requiring little maintenance, being able to operate continuously for periods of weeks or months, and causing minimal or no disturbance to visitors (Watson et al. 2000). A survey of land managers in the U.S.A. including 169 state land managers and 175 federal land managers found $55.2 \%$ of state land managers and $73.3 \%$ of federal land managers use mechanical/electronic counting devices to gauge visitor use on trails. Furthermore, about 75\% of these land managers cited using these data for future planning and capital projects (Lynch et al. 2002).

Despite relatively widespread reliance on automated visitor monitors to estimate recreation visitation, previous research suggests that land managers consider the devices to be ineffective because of the difficulties associated with installation of the units and calibration of the recorded data (Lynch et al. 2002). Several other studies have documented, empirically, the fact that there is some amount of error associated with all visitation data collected via automated visitor monitors (Bates, Wallace, \& Vaske, 2007; Gracia-Longares, 2005; Lindsey et al. 2006; Muhar, Arnberger, \& Brandenburg 2002). Factors that contribute to data error include the physical aspects of the monitoring locations (e.g., trail slope and width). For example, on a wide trail, people walking side by side in groups increases the chances of not all visitors being detected by a mechanical counter. In contrast, narrow trails force people to walk single file and 
pass a counter one at a time, increasing the chances that all passing visitors will be detected by the monitor. Likewise, monitors placed in areas where visitors tend to pause, such as overlooks and trail junctions, will count an individual multiple times if they pause within the counter's range of detection. Automated visitor monitors are also subject to error resulting from climatic conditions such as heavy snow, blowing vegetation, visitor tampering, and/or direct sunlight striking the monitor’s “receiving eye” at sunrise or dusk (Vaske, Shelby, \& Donnelly, 2008). Thus, raw data from automated counters cannot be treated as reliable and accurate measures of visitor use. Rather, automated visitor counters must be calibrated to estimate data error and convert raw counter data to reliable estimates of visitation.

Despite the prevalence of studies involving the use of automated visitor monitors and the inherent error in visitor use counts recorded with these devices, few studies have documented specific calibration methods to convert raw data to reliable visitation estimates. Of those studies that do document calibration methods, Watson et al. (2000) provide the most thorough description, including discussion of sampling methods to calibrate monitors and basic statistical analyses to estimate visitor use from automated counter data. The authors describe observerbased counting techniques to correct automated monitor counting errors but do not suggest observation lengths for each observation sampling period (e.g., 30 minutes vs. one hour). Likewise, Bates et al. (2006) used infrared monitors to estimate visitor use in Rocky Mountain National Park (NP), U.S.A. and conducted observation-based calibration counts over three days during the 2004 summer season. However, the authors do not describe the methods by which they selected the number of observation sampling days and duration of observation sampling periods. Both studies (Watson et al., 2000; Bates et al., 2006) conclude that it is necessary to calibrate automated visitor counters through direct observations in order to calculate accurate 
estimates of visitor use. However, neither study provides sufficient detail about calibration methods to allow for their procedures to be replicated.

Based on the studies previously described, automated visitor counters are typically calibrated through human observation. However, certain precautions need to be accounted for in the data collection protocol to ensure that counts are accurate. Specifically, previous research suggests the number of variables collected by an observer effects data accuracy. For example, Arnberger, Haider, and Brandenburg (2005) collected various data about visitor use including: 1) number of visitors; 2) type of user group; 3) group size; 4) direction of travel; 5) number of dogs off leash; and 6) and time of visit. These data were collected by human observers and video recording devices simultaneously in order to test the accuracy of counts collected by humans observers versus electronic video observation. Results from this study indicate that at high levels of overall use (>120 pph) field observers reported $20 \%$ less visitors compared to video interpreters. In a similar study, Greene-Roesel, Diogenes, Ragland, and Lindau (2008) collected pedestrian counts through direct human observation and electronic video observation to calibrate automated pedestrian counters. Observers only collected overall visitor use counts (i.e. no distinctions were made to identify direction of travel). Results indicated little difference between human observations and video observations (-0.9\% to $1.4 \%)$ even during periods of high visitor use (e.g. 654 people per hour). These studies suggest that data collection protocols need to minimize the number of variables collected by an observer to ensure reliable counts derived through human observation.

Several studies have examined correlations between observed counts and those recorded via automated visitor monitors, and those studies have generally reported strong correlations. For example, Muhar et al. (2002) present results that show a very strong correlation between monitor 
counts and observed counts $\left(R^{2}=0.94\right)$, but no details of observation methods to correct monitor counting errors are provided. Lindsey et al. (2006) used automated visitor monitors to estimate visitor use on urban trails in Indianapolis, Indiana U.S.A over a four year period. Within the study, infrared monitors were installed at 28 locations to conduct automated counts of visitor use. In addition, a total of 442 hours of direct observation counts were conducted at the 28 locations where infrared monitors were operating. The observation and automated monitor data were aggregated for the 28 locations, and regression analyses were conducted to estimate a correction factor to apply to data recorded by all 28 automated monitors. While the estimated correlation between observation and automated monitor data was very high $\left(R^{2}=0.99\right)$, the standard error associated with the correction factor was not estimated.

Gracia-Longares (2005) used automated visitor monitors to estimate visitor use in Yellowstone NP, U.S.A. and examined the effects of the duration of observation-based calibration counting periods on the precision of corresponding estimated correction factors. In particular, correlation coefficients were estimated based on 4-hour, 1-hour, 30 minute, and 15 minute observation-based calibration counting periods (each totaling four hours of observation i.e., a single 4-hour period, four 1-hour periods, eight 30-minute periods, and 16 15-minute periods). Correlation coefficients were highest for the data collected via 4-hour sampling periods and decreased as the duration of the observation period decreased. The largest decrease in the correlation between observed counts and those recorded with the automated visitor monitors occurred between the 1-hour observations and the 30 minute observations. Moreover, while data from all observation lengths resulted in similar correction factors, the precision of correlation estimates improved with data based on longer observation periods. These results suggest that sampling periods for observation-based calibration counts should be at least one hour in duration. 
However, all of the study sites were relatively low use areas ( $<75$ people / day) and the conclusions regarding sampling period duration may not apply in higher use areas.

In some cases, errors contained within automated visitor monitor data are sufficiently excessive that they can be relatively easy to detect from visual inspection and should be excluded from the dataset prior to analyses. For example, automated visitor monitors have been reported to malfunction, causing the devices to record an inordinately large number of pass-by events; in other case, devices malfunction and record no pass-by events during periods of time and in locations where substantive levels of visitation are expected. One method for treating data errors of this nature is to estimate counts for missing or otherwise obviously erroneous data from known automated visitor monitor counts with similar characteristics. For example, missing data from a given Tuesday can be estimated from the previous and following Tuesday counts (Lindsey \& Nguyen, 2004; Vaske et al. 2008). This method of substitution simplifies calculations of population parameters because all data from the population are accounted for and assumes no errors in parameter estimates. However, this method may not be appropriate for datasets containing more than a few occurrences of missing or otherwise obviously erroneous data because population parameter estimates may result in misleading estimates of statistical precision (Scheaffer, Mendanhall III, \& Ott, 2006).

In summary, the use of automated visitor monitors for estimating recreational visitation in protected natural areas is relatively common, yet data from such devices are subject to several sources of error. Consequently, raw data from automated visitor monitors universally require some form of calibration to generate valid and reliable estimates of visitor use. However, few published studies have comprehensively documented methodological procedures and/or empirical results for calibrating recreational visitation data recorded with automated visitor 
monitors. Consequently, standardized methods to calibrate automated visitor monitors are yet to be established. The purpose of this paper is to document systematic sampling and analysis methods for collecting and calibrating visitor use data recorded via automated visitor monitoring devices. Specifically, this paper describes the collection and calibration of automated counter data to estimate current visitor use levels at popular attraction areas within the Merced Wild and Scenic River watershed in Yosemite NP, U.S.A.

\section{Sampling Methods}

\subsection{Study Location}

Yosemite NP is centrally located in the Sierra Nevada mountain range in California, U.S.A. and protects 285,151 hectares of rugged mountain terrain. Yosemite NP has some of the most spectacular scenery in the National Park Service (NPS) and views of glacier carved valleys can be seen from many locations throughout the park. People from around the world come to experience Yosemite NP and visitation reached 3.5 million visitors in 2007 (NPS, 2008).

Yosemite Valley is the most popular destination area in the park, offering iconic views of glacial features, including El Capitan and Half Dome. The Merced River bisects the north and south sides of Yosemite Valley and is designated a Wild and Scenic River in order to protect its free-flowing condition and unique values (NPS, 2005). Vehicle access is along a one-way road

that enters from the west end of Yosemite Valley and follows the south side of the Merced River. About $90 \%$ of visitors to Yosemite NP arrive by automobile and $87 \%$ of visitors cite "taking a scenic drive” as their favorite activity in the park (White \& Aquino, 2008).

The design and layout of roads, parking, and facilities within Yosemite Valley were never meant to accommodate the intensive amount of vehicle traffic they receive today. Consequently, traffic congestion in Yosemite Valley is common during periods of peak 
visitation. For example, on a typical day between May and September, up to 1,900 day use vehicles at a time are in Yosemite Valley competing for approximately 1,200 designated day-use parking spaces. During the summer, the main day-use visitor parking area fills to capacity between 11:00 am and 1:00 pm. As a result, visitor use at attraction sites during peak hours is very high, posing potential threats to the integrity of park resources and the quality of visitors' experiences. Consequently, the NPS requires precise information about visitation to key venues within Yosemite Valley to inform decisions about how to manage visitor use in a manner that is consistent with resource protection and visitor experience objectives.

\subsection{Automated Visitor Monitor Equipment}

TrailMaster TM1550 active infrared monitors were deployed to estimate visitor use at selected attraction sites and trailheads in Yosemite Valley. These devices are designed to monitor and study wildlife, but have also been successfully applied to recreation use monitoring in protected natural areas (Bates et al., 2006; Gracia-Longares, 2005; Vaske \& Donnelly, 2007). The TrailMaster TM1550 monitor system is comprised of a transmitter and a receiver placed on opposite sides of a trail. Infrared energy is emitted from the transmitter in short pulses and is detected by the receiver. The receiver registers a count when the infrared beam is interrupted by the presence of a physical object, such as a hiker. The TM1550 model used in this study stores up to 16,000 pass-by events, with each recorded event containing a date and time stamp accurate to the minute. A pass-by event is defined here as any event (i.e., a hiker that passes between the monitor transmitter and receiver regardless of the direction of travel) that triggers a count on the infrared monitoring device. The study monitors stop registering time stamped events when their memory reaches capacity (i.e., when 16,000 counter events are registered on a single unit). A TrailMaster DataCollector is used to download data from the monitors in the field and to upload 
data to a personal computer in an office environment. With each data download, the counter's memory is cleared by deleting the existing data and reset to its maximum storage capacity (i.e., 16,000 counter events) for the next monitoring period. Within this study, the time interval between data downloads was scheduled individually for each counter based on the relative intensity of use at each counter location (i.e., the expected number of days by which the memory capacity would be reached).

\subsection{Automated Visitor Monitor Locations}

Because of the paucity of information about calibration techniques within existing literature, a pilot study was conducted to estimate the number of sampling hours that would be required to collect the necessary amount of observation data needed to calibrate data from the automated visitor monitors with a sufficient level of precision. We anticipated that the accuracy and associated calibration sampling effort required for the automated visitor monitors would vary as a function of the level of visitor use at each monitoring location. In particular, we expected monitor counts to be less accurate in locations with higher levels of use, where it is common for multiple visitors to pass-by the monitoring device simultaneously. Thus, we conducted the pilot study on the Yosemite Falls trail because visitor use changes dramatically throughout the day and we consequently expected the location would provide a comprehensive evaluation of data requirements for calibration under a range of visitor use levels. The site was also selected for the pilot study because it contains trail segments of varying widths, thus providing an empirical basis to assess calibration sampling effort requirements for a range of trail widths. Therefore, data collection for the pilot study involved placing an automated visitor monitor proximate to each of the two access points to Lower Yosemite Falls. 
Implementation of the full study involved purposively placing automated visitor monitors at popular attraction sites and trailheads in Yosemite Valley, selected by researchers in consultation with Yosemite NP staff (Figure 1 and Table 1). Several factors were considered in the process of selecting specific locations at which to install the monitors. In particular, narrow trail sections were selected, as they are places where visitors are most likely to walk single file. Locations were also selected where there was a straight and clear line of sight between transmitter and receiver to ensure a strong signal between the components. Selected monitoring locations also provided some form of cover where the automated visitor monitors could be hidden from trail users so as not to degrade the visitor experience and/or increase the likelihood of vandalism to the monitors. Finally, monitors were installed in locations away from trail junctions or areas of interest, as these are locations where visitors tend to pause and could consequently be counted multiple times by the monitoring devices.

\section{Table 1}

Locations of study sites where automated visitor monitors were installed

\begin{tabular}{clcc}
\hline $\begin{array}{c}\text { Monitor } \\
\text { Number }\end{array}$ & \multicolumn{1}{c}{ Location } & Use Level & Date Placed in \\
\hline 1 & Yosemite Falls (West) & High & May 19, 2007 \\
2 & Yosemite Falls (East) & High & May 19, 2007 \\
3 & Vernal Fall Trailhead & High & May 23, 2007 \\
4 & Bridalveil Fall & High & May 24, 2007 \\
5 & Glacier Point (Main Trail) & High & June 2, 2007 \\
6 & Glacier Point (Handicap Accessible Trail) & Low & June 25, 2007 \\
& & & \\
\hline
\end{tabular}




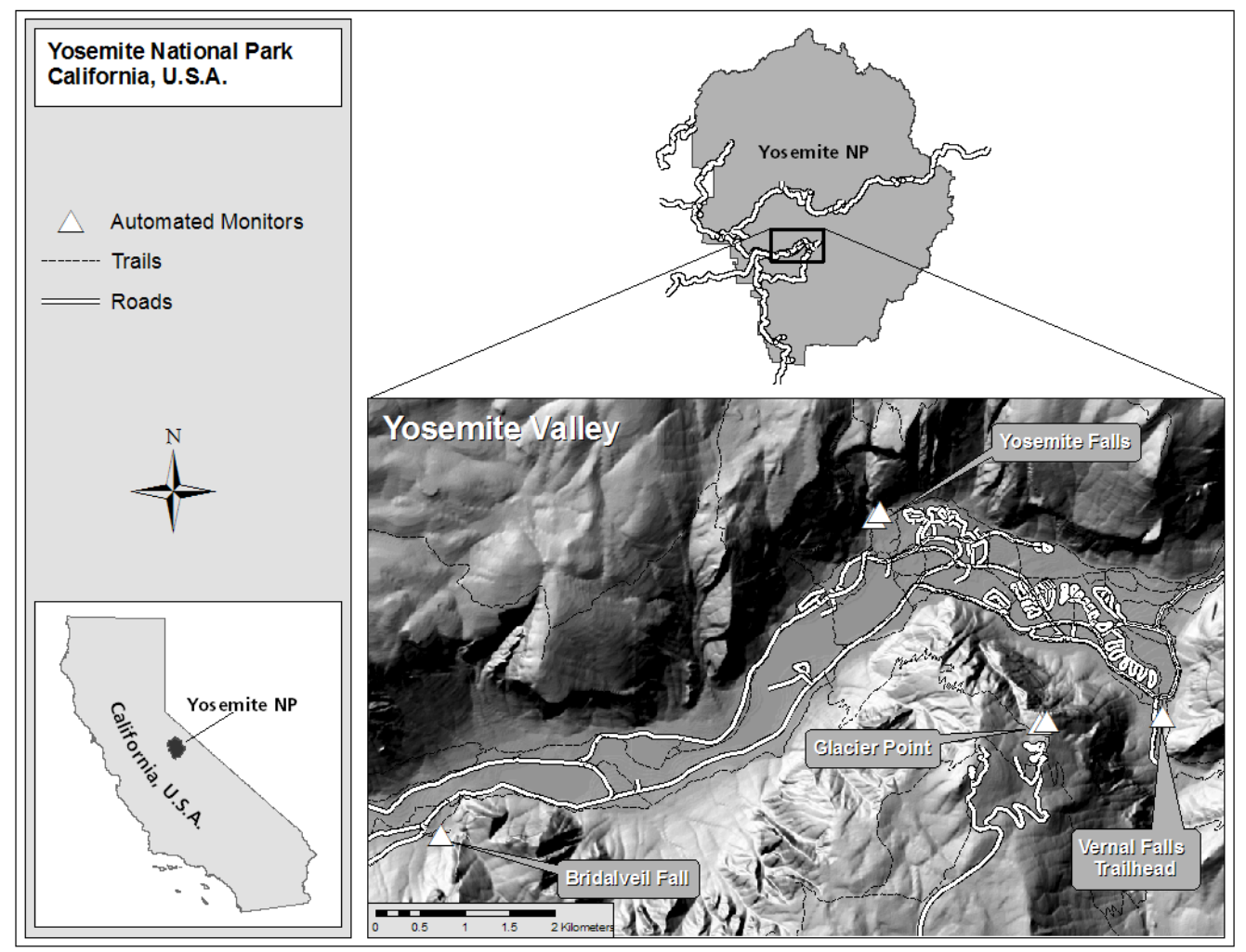

Figure 1. Location of automated counters on the trail to Yosemite Falls

\subsection{Direct Observation of Visitor Use at Monitoring Locations}

As stated, raw data collected via automated visitor monitors were calibrated using observation-based visitor count data collected at the monitoring locations during a sample of days. A primary purpose of the pilot study on the Yosemite Falls trail was to assess the number of hours of observation data required to generate sufficiently precise calibrations of and corresponding estimates of visitation from the automated visitor monitor data. Within the pilot study, nine one-hour observations of visitor use were conducted from May 20 to May 22, 2007 proximate to the two automated visitor monitor locations on the trail to Lower Yosemite Falls (Figure 2). Starting times for each of the nine observation periods were randomly selected, ranged from 7 am to $6 \mathrm{pm}$, and occurred on the hour. During each observation sampling period, 


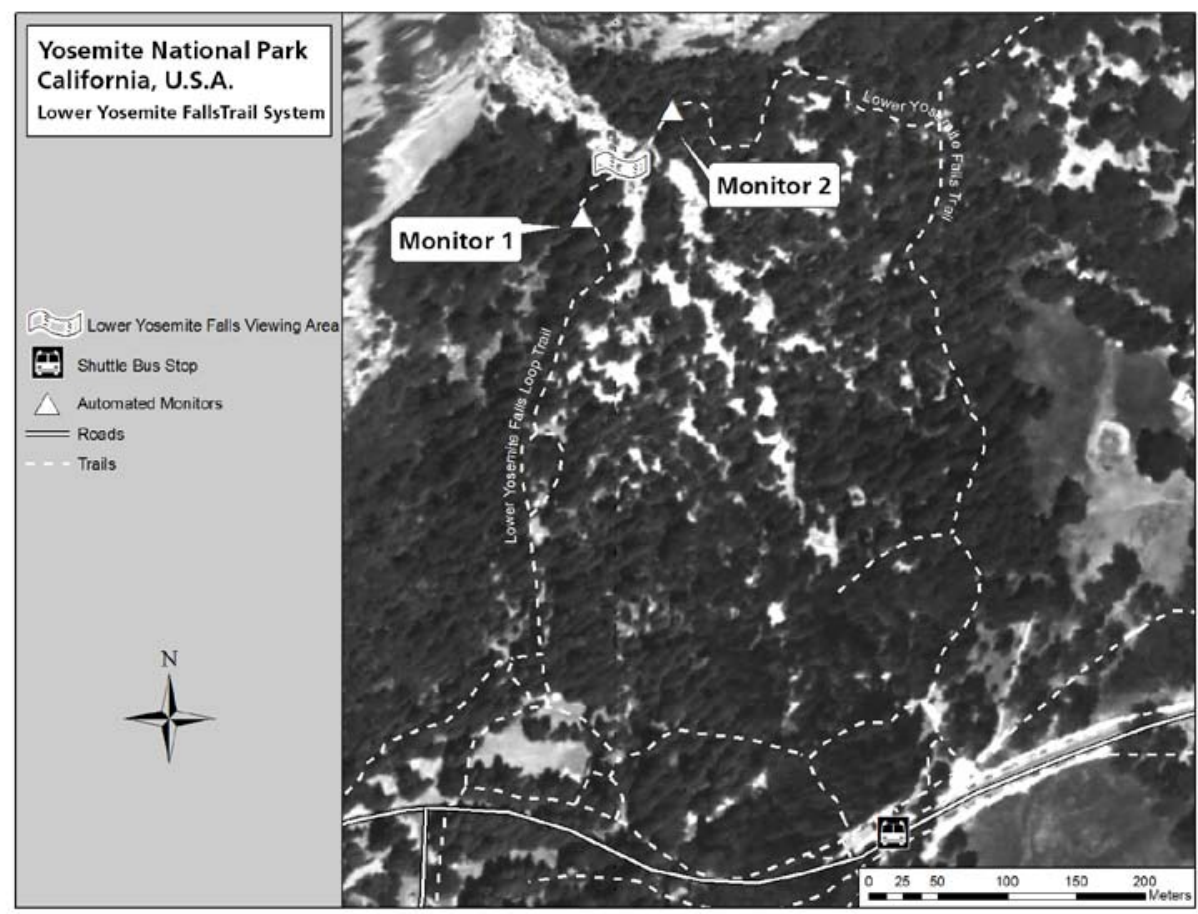

Figure 2. Locations of Yosemite Valley visitor monitors

one observer was stationed proximate to each of the two automated monitors to assess each device's performance and collect calibration data (i.e., direct observation of the number of visitor pass-bys and visitors' direction of travel). Data collection was limited to 2 variables (i.e. visitor arrivals and departures) to ensure the data accuracy. In addition, observers were provided with thumb counters (one for arrivals and one for departures) to ensure that observers could visually focus on visitors as they passed the automated monitor. The observers recorded data in 15minute intervals on a standardized form that included entries for: 1) observed visitor arrival and departure counts; and 2) automated visitor monitor counts recorded at the beginning and end of each observation period (i.e., the number of pass-bys recorded by the monitor during the hour of observation-based counting). 
The observation data were used in a two-step process to calibrate visitor pass-by data recorded by the automated visitor monitors. First, the observation data were used to correct for error in the devices' detection of pass-by events, as described in the following section entitled “Analytical Methods”. Second, the observation data were used to scale the calibrated pass-by events, which include events for arriving and departing visitors, to estimates of the number of calibrated pass-by events associated with arriving visitors (i.e., site visitation). The scaling procedure takes into account changes in the proportions of arriving and departing visitors, by time of day. For example, at some study sites there is a greater proportion of arriving than departing visitors during the morning hours, and a greater proportion of departing than arriving visitors later in the day.

\section{Analytical Methods}

Several analytical steps were conducted to convert raw data recorded via automated visitor monitors to precise estimates of visitor use at the study sites in Yosemite Valley. In particular, the pilot study data from the Yosemite Falls trail were analyzed to estimate the direct observation sampling effort required to provide sufficiently precise calibrations of the automated visitor monitor data collected at the study sites. Second, bootstrap techniques were used to account for missing automated monitor data and estimate mean pass-by events per hour, day, month, and season for each monitoring device. Third, regression analyses were performed to estimate calibration or correction factors for the automated visitor monitor data from each site, based on the visitor use count data (i.e., calibration data) collected via direct observation. Fourth, correction factors were applied to the bootstrap-estimated mean pass-by events per hour, day, month, and season. Fifth, the calibrated mean pass-by results were scaled to arrivals based on the 
proportion of arrivals versus departures, by time of day, recorded via direct observation. The following sections of the paper describe these analytical methods in detail.

\subsection{Estimation of Direct Observation Sampling Effort Required for Precise Calibration}

As stated, a primary purpose of the pilot study on the Yosemite Falls trail was to estimate the number of hours of calibration data needed to produce sufficiently precise estimates of visitation for the study sites. Results of the pilot study were used in equation 1 to estimate required calibration sample size (i.e., hours of observation data required) to achieve sufficiently precise calibrations of the automated visitor monitor data (Scheaffer et al. 2006).

$$
n=\frac{N \sigma^{2}}{N D+\sigma^{2}}
$$

Where:

$n=$ required sample size (number of hourly observation periods)

$N=$ total number of hourly observation periods during study

$\sigma^{2}=$ variance (estimated from the pilot study)

$B=$ error of average hourly visitor use estimation

$D=B^{2} / 4$

From these equations, the number of hours required to achieve 1\%, 2\%, 3\%, $4 \%$ and 5\% error were estimated (Table 2). Due to the limitations of scheduling and travel time between study sites 24 hours of direct observations were chosen to be conducted at each study site. Twenty-four hours of direct observations were estimated to yield an error of approximately 3.5\% at unit 2 and $4.5 \%$ at unit 3 and were used as a baseline for all study sites. For each study site, six direct observation sampling days were randomly selected between June 1 - August 31 and four one-hour periods within each selected sampling day between 7 am - 6 pm during which to conduct direct observations were randomly selected. Of note, only 16 hours of observations were conducted at unit 6 because this site was not considered a priority and due to equipment 
availability an automated visitor monitor was not installed at this site. However, an automated visitor monitor became available in late June and was installed at this time. The sampling schedule for this was coordinated with unit 5 and resulted in 16 hours of direct observations.

\section{Table 2}

Estimated Number of Sampling Hours Required at Yosemite Falls

\begin{tabular}{ccc}
\hline Monitor Location & Percent Error & Number of Sampling Hours \\
\hline 2 & $5 \%$ & 10 \\
2 & $4 \%$ & 26 \\
2 & $3 \%$ & 59 \\
2 & $2 \%$ & 205 \\
3 & $1 \%$ & 20 \\
3 & $5 \%$ & 31 \\
3 & $4 \%$ & 54 \\
3 & $3 \%$ & 117 \\
3 & $2 \%$ & 393 \\
\hline
\end{tabular}

\subsection{Bootstrap Analysis to Account for Missing Automated Visitor Monitor Data}

Following sampling at all study locations, data from the automated visitor monitors were uploaded to a personal computer using TrailMaster Stat Pack software. Data were collected from the mechanical counters between June 1 and September 30, 2007. The data were imported into the database program Microsoft Access and queried using structured query language (SQL) to aggregate the time-stamped data from the automated visitor monitors into: 1) total pass-by events recorded during the sampling season (June 1-September 30); 2) pass-by events per month; 3) 
pass-by events per day, by day of the week and month; and 4) pass-by events per hour. However, some counter data were lost during the season due to operating issues (e.g., a counter's memory reached capacity before research technicians were able to conduct a data download), thus total and mean pass-by events computed based on the raw data are biased due to missing data.

Examination of the raw data from the automated visitor monitors revealed most of the data to be positively skewed, thus, classic statistical methods were not appropriate to estimate population parameters. Therefore, we used bootstrapping methods to estimate mean pass-by events, by period of time (i.e., hour, day, month, season), to account for the fact that all of the monitors had some instances of missing data.

Bootstrapping is a resampling method to make statistical inferences from a distribution that approximates the distribution of a population (Crawley, 2005; Scheaffer et al. 2006). In this case, the approximating distributions are the incomplete datasets of monitor counts and the population distributions are the corresponding complete sets of monitor counts that would have resulted without device operating issues and other sources of missing data. The approximating distribution is randomly sampled with replacement at sample size $n$ from the original sample of size $n$ many times (i.e., $1,000+$ times) to estimate statistical parameters for the population distribution (mean hourly, daily, and seasonal pass-bys in this case). A distribution of means is obtained from the $1,000+$ samples and the mean of this distribution is the estimated mean for the population.

We created a script using the ' $R$ ' statistical program to resample the raw data for each monitor 10,000 times to estimate mean hourly, daily, monthly, and summer season pass-bys for each monitor. For study sites with multiple monitors, however, data from each monitor at the 
study site were summed and bootstrapping was performed on the combined data to estimate mean hourly, daily, monthly, and summer season pass-by events, in total, at the study site.

\subsection{Calculating Correction Factors}

Mean hourly pass-bys recorded by the automated monitors during calibration sampling were paired with corresponding mean hourly pass-bys counted through direct observation to estimate correction factors for each automated monitor. The specific methods used to estimate correction factors for monitors at study sites with a single monitoring location differed from those used at study sites with multiple monitoring locations, as described in the following sections.

\subsection{Study Sites with a Single Monitoring Location}

Two of the study sites, Vernal Fall and Bridalveil Fall, each required just one monitoring location from which to record visitor pass-bys as a basis to estimate site visitation. For each of these two study sites with a single monitoring location, linear regression modeling was used to estimate a correction factor $(r)$ for the automated visitor monitor to convert bootstrap-estimated mean hourly, daily, monthly, and summer season pass-bys to calibrated estimates of mean hourly, daily, monthly, and summer season pass-bys. Each linear regression model (i.e., one for each automated visitor monitor) was specified with the hourly visitor pass-bys recorded by the automated visitor monitor during calibration sampling hours entered as the independent variable and the corresponding observation-based counts of hourly visitor pass-bys specified as the dependent variable. Thus, for monitors 1-5, there were 24 pairs of automated monitor and direct observation counts entered into the regression model, and 16 pairs of counts entered into the regression model for monitor 6. Further, in keeping with ratio estimation techniques, each regression model was specified with no constant term, to force the regression line through the 
origin. Within each regression model, the estimated regression coefficient for the independent variable serves as the correction factor $(r)$ for converting bootstrap-estimated mean hourly, daily, monthly, and summer season pass-bys from the corresponding automated visitor monitor to calibrated estimates of mean hourly, daily, monthly, and summer season pass-bys.

\subsection{Study Sites with Multiple Monitoring Locations}

Two of the study sites, Yosemite Falls and Glacier Point, each required more than one monitoring location from which to record visitor pass-bys as a basis to estimate site visitation. For several reasons, the methods for estimating correction factors for automated visitor monitors at single-monitor sites are not sufficient to estimate correction factors at multiple-monitor sites. In particular, each automated visitor monitor, whether it be at a single-monitor or multiplemonitor site, has a unique correction factor $(r)$ and standard error of $r\left(S E_{r}\right)$ value. Moreover, the relative amount of use varies at each access point within the two multiple-monitor sites; consequently, visitor pass-by counts from each monitor have differently weighted contributions to estimates of mean and total site visitation. For these reasons, it was necessary to adapt singlemonitor site regression modeling methods, as described in the following paragraph, to estimate weighted values of $r$ and $S E_{r}$ for each automated monitor within each multiple-monitor site, based on the relative number of pass-by events recorded by each monitor.

For each of the two study sites with multiple monitoring locations, regression models were estimated separately for each automated visitor monitor. Each linear regression model (i.e., one for each automated visitor monitor) was specified with the hourly visitor pass-bys recorded by the automated visitor monitor during calibration sampling hours entered as the independent variable and the corresponding observation-based counts of hourly visitor pass-bys specified as the dependent variable. Further, in keeping with ratio estimation techniques, each regression 
model was specified with no constant term, to force the regression line through the origin.

Within each regression model, the estimated regression coefficient for the independent variable serves as the correction factor $(r)$ for the corresponding automated visitor monitor. In the case of the multiple-monitor sites, the estimated correction factor for each automated visitor monitor was entered into a pair of equations to compute a composite correction factor and standard error, based on weighting regression results from each monitor. Study data from Yosemite Falls are used in the following equations 2-3 to demonstrate the procedures used to estimate weighted calibration factors for automated visitor monitors within multiple-monitor study sites.

$$
\begin{aligned}
& r_{\text {combined }}=\frac{\left(r_{\text {unit } 1} \cdot \hat{\tau}_{\text {unit } 1}\right)+\left(r_{\text {unit } 2} \cdot \hat{\tau}_{\text {unit } 2}\right)}{\hat{\tau}_{\text {combined }}}=\frac{(1.83125 \cdot 228,831)+(1.66902 \cdot 138,689)}{367,520}=1.77003 \\
& S E_{r(\text { combined })}=\frac{\left(S E_{r(\text { unit } 1)} \cdot \hat{\tau}_{\text {unit } 1}\right)+\left(S E_{r(\text { unit } 2)} \cdot \hat{\tau}_{\text {unit } 2}\right)}{\hat{\tau}_{\text {combined }}}=\frac{(.01808 \cdot 228,831)+(.01704 \cdot 138,689)}{367,520}=.01769
\end{aligned}
$$

\subsection{Calculating Visitor Use from Monitor and Calibrations}

Correction factors estimated via regression analyses and bootstrap estimates of mean hourly, daily, monthly, and summer season pass-bys for each study site were used in the following formulae to estimate calibrated mean hourly, daily, monthly, and summer season passbys at each study site (equations 4-8). In the case of multiple monitor sites (i.e., Yosemite Falls and Glacier Point), the composite correction factor and standard error are used in the equations to estimate the calibrated mean number of pass-bys at the study site, by period of time. 


$$
\begin{aligned}
& \hat{X}=(r) \cdot(\hat{x}) \\
& \operatorname{Var}(r)=[S E(r)]^{2} \\
& \operatorname{Var}(\hat{X})=(\hat{X})^{2} \cdot \operatorname{Var}(r) \\
& B(\hat{X})=2 \cdot \sqrt{\operatorname{Var}(\hat{X})} \\
& \begin{aligned}
& C I(\hat{X})=\hat{X} \pm B \\
& \text { Where: } \\
& \hat{X} \quad=\text { calibrated mean (hourly, daily, monthly, or summer season) pass-bys } \\
& r \quad=\text { correction factor (composite value in the case of multiple monitor sites) } \\
& \hat{X} \quad=\text { bootstrap-estimated mean (hourly, daily, monthly, or summer season) pass-bys } \\
& \operatorname{Var} \quad=\text { variance of the correction factor } \\
& S E \quad=\text { standard error of the correction factor (composite value in the case of multiple monitor sites) } \\
& B \quad=\text { bounds of estimation } \\
& C I \quad=\text { confidence interval }
\end{aligned}
\end{aligned}
$$

Finally, data concerning visitors’ direction of travel recorded in a companion study conducted in Yosemite Valley (Lawson et al. 2008) were used to convert calibrated mean hourly, daily, monthly, and summer season pass-bys to estimates of hourly, daily, monthly, and summer season visitation. In particular, estimates from the companion study of the proportion of pass-bys constituting visitor arrivals (rather than departures), by hour of the day, were applied to the calibrated mean hourly pass-bys to estimate mean hourly visitation to each study site. To estimate daily, monthly, and summer season visitation, the corresponding calibrated mean number of pass-bys was multiplied by 0.5 , because all study sites are day use sites and consequently all arriving visitors are assumed to depart at some time during the day.

\section{Results}

\subsection{Monitor Calibration}

At all study sites, monitor counts were highly correlated with observed counts. Scatterplots of observed counts and monitor counts at Bridalveil Fall (Unit 4) and Glacier Point 
(Unit 5) demonstrate this strong relationship (Figure 3). The same strong relationship between observed and monitor counts was found at all of the other study sites.
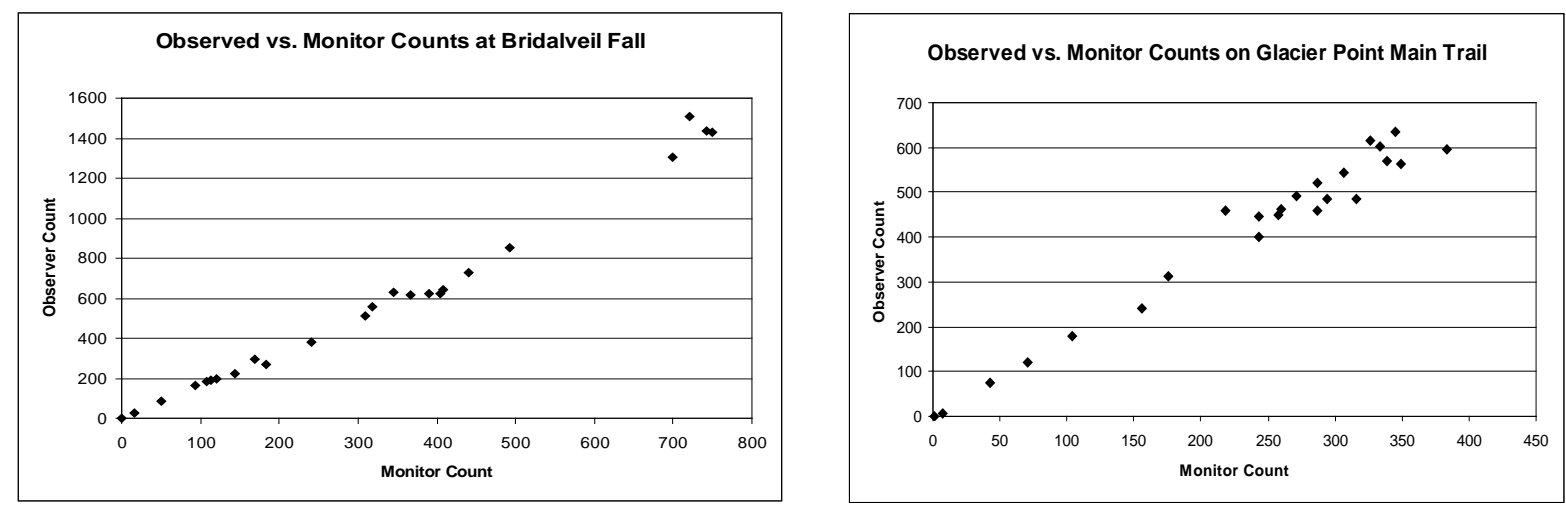

Figure 3. Scatterplots of observed versus monitor counts for the Bridalveil Fall trail and Glacier Point main trail

The scatterplots of observed counts versus monitor counts confirmed our choice to use ratio estimation techniques to calibrate monitor data. For example, direct observation-based and automated visitor monitor counts of visitor pass-bys plotted in Figure 3 shows these variables to be highly correlated at all levels of use, pass through the origin, and are slightly more variable at higher levels of use (Scheaffer et al., 2006). Thus, simple linear regression models with the regression line forced through the origin were estimated and produced very strong model fits (Table 3). 


\section{Table 3}

Regression analysis results with regression line forced through origin for all automated monitors

\begin{tabular}{ccccccc}
\hline Unit & $\beta^{*}$ & $S E$ & $R^{2}$ & $d f$ & $F$ & $p$ \\
\hline 1 & 1.83125 & .01808 & .9977 & 1,23 & 10,025 & $<.001$ \\
2 & 1.66902 & .01704 & .9976 & 1,23 & 9,588 & $<.001$ \\
3 & 1.70400 & .01900 & .9973 & 1,22 & 8,036 & $<.001$ \\
4 & 1.82560 & .03570 & .9917 & 1,22 & 2,615 & $<.001$ \\
5 & 1.72431 & .02707 & .9944 & 1,23 & 4,057 & $<.001$ \\
6 & 1.56545 & .08539 & .9600 & 1,15 & 336 & $<.001$ \\
\hline
\end{tabular}

* The regression coefficient $(\beta)$ constitutes the correction factor $(r)$ for the corresponding monitor.

\subsection{Estimated Use}

This section of the paper presents estimates of seasonal visitation for each study site.

Data from units 1 and 2 were combined to estimate use at Yosemite Falls and data from units 5 and 6 were combined to estimate use at Glacier Point. Hourly visitation results for these two study sites are presented in Figure 4. These estimates show that visitor use at Yosemite Falls varies by month, day of week, and time of day. 

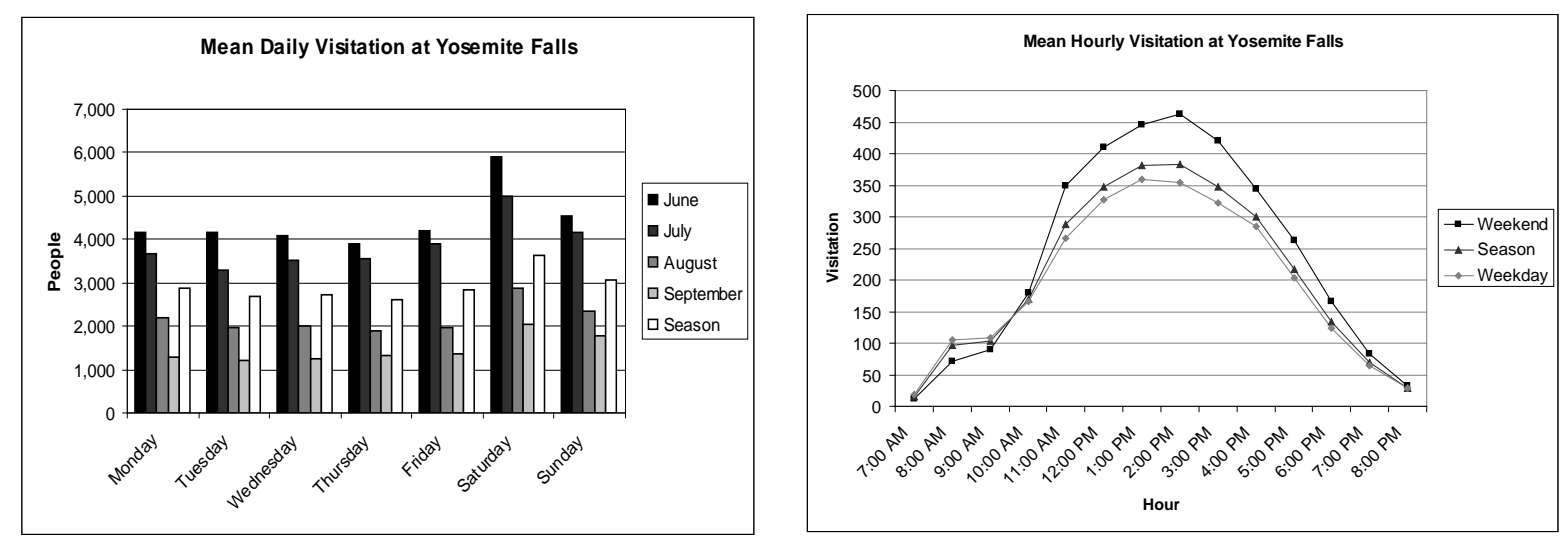

Figure 4. Seasonal estimates for mean daily and mean hourly visitation at Yosemite Falls

Daily visitor use is presented in Table 4. Bootstrapping techniques were not used to estimate use for Tuesday, Wednesday, and Thursday because all data were collected for those days and population parameters did not need to be estimated. Visitor use is similar during weekdays but is substantially higher on Saturdays.

\section{Table 4}

Mean daily visitation at Yosemite Falls (Season)

\begin{tabular}{|c|c|c|c|}
\hline Day & Total Number of Days & $\begin{array}{c}\text { Number of Days } \\
\text { with Data }\end{array}$ & Mean \\
\hline Monday & 17 & 14 & $2,857 \pm 57$ \\
\hline Tuesday & 17 & 17 & $2,693 \pm 54$ \\
\hline Wednesday & 17 & 17 & $2,717 \pm 54$ \\
\hline Thursday & 17 & 17 & $2,625 \pm 52$ \\
\hline Friday & 18 & 17 & $2,827 \pm 56$ \\
\hline Saturday & 18 & 15 & $3,633 \pm 73$ \\
\hline Sunday & 18 & 15 & $3,075 \pm 61$ \\
\hline
\end{tabular}


Finally, calibrated seasonal visitor use totals estimated from bootstrapping are presented in Table 5. Visitor use during the summer months in Yosemite is very high with over 300,000 people visiting Yosemite Falls and Glacier Point between June and September. In addition, note the high level of precision of the estimates.

\section{Table 5}

Total Estimated Number of People at each Study Location (June 1-September 30)

\begin{tabular}{cccc}
\hline Unit/ Location & $\begin{array}{c}\text { Total Number of Days } \\
\text { in Study Period }\end{array}$ & $\begin{array}{c}\text { Number of Days } \\
\text { with Data }\end{array}$ & Total \\
\hline 1 & 122 & 118 & $233,335 \pm 4,607$ \\
3 & 122 & 112 & $126,040 \pm 2,574$ \\
4 & 122 & 92 & $246,606 \pm 5,499$ \\
5 & 122 & 105 & $331,862 \pm 10,420$ \\
6 & 122 & 99 & $5,145 \pm 561$ \\
Yosemite Falls & 122 & 82 & $354,423 \pm 7,083$ \\
Glacier Point & 122 & 112 & $346,500 \pm 11,273$ \\
\hline
\end{tabular}

\section{Discussion}

This study shows that automated visitor counters produce data from which reliable estimates of visitor use can be calculated. These results provide important insights for researchers and park managers using these devices to estimate visitor use in parks and protected areas. Moreover, the methods described in this paper provide a comprehensive system to collect 
observation data to calibrate automated visitor counters and produce accurate estimates of visitor use.

\subsection{Monitor Calibration}

At all study sites, automated monitor counts were highly correlated to observation based counts. Regression models calculated from these data produced $R^{2}$ values greater than 0.95 and significant $p$-values indicate that all regression coefficients $(r)$ were statistically significant. These results show that automated raw monitor counts need to be corrected to obtain accurate estimations of visitor use. Moreover, each automated visitor counter had a unique $r$ and $S E_{r}$ value suggesting that monitor error is related to how groups of hikers align themselves along the trail based on local conditions such as slope, trail width, and trail condition and not related to the overall quantity of use. For example, visitors may walk in a group formation on wide trail sections while narrow trail sections dictate that groups walk side by side or in single file.

Consistent with Gracia-Longares (2005), we found our correction factors became less variable as the observation period increased. Large amounts of visitor traffic were often undercounted during short periods of time (15 minutes or less), however, these errors were generally offset over the course of an hour by periods when individuals and small groups would be accurately captured by the monitor. Similarly, people walking side by side during low use periods only triggered a single count. Again, these errors tended to even out over the course of an hour.

\subsection{Treatment of Missing Data}

Bootstrapping was found to be an effective method for treating missing data and offers three advantages over replacing missing values with means. Bootstrapping does not require a set of rules to determine how mean values are obtained. Typically, mean values to replace missing 
data are calculated from the same day of the week from the previous and following weeks of a missing count. However, there are potential shortcomings to replacing missing values with means. For example, holidays can affect the results of these calculations or force the researcher to modify the rules from which the mean is obtained (e.g., the Monday before or after Labor Day). In addition, replacing missing data with mean values may not be possible for datasets with more than a few missing points because days from which means are calculated may be missing. Furthermore, bootstrapping is robust to the amount of missing data because population parameters can be estimated from datasets missing more than a few data points.

\subsection{Other Automated Counting Systems}

Since the time this study was conducted the authors have had the opportunity to use other brands of automated visitor counting devices including EcoCounter (Lannion, France) and TRAFx counters (Canmore, Canada) to collect visitor use data at other locations in Yosemite NP. Similar to the TrailMaster counter used in this study, the EcoCounter and TRAFx counters use active infrared technology, however, these counters offer some advantages over the TrailMaster model. The EcoCounter is able to detect the direction of travel of a hiker (i.e. this device distinguishes between arriving and departing hikers) and separate records are kept of arriving, departing, and total hikers. In addition, the memory capacity of the EcoCounter is much larger than the TrailMaster counter. The manufacturer does not provide a memory capacity but based on the device's screen counts its memory capacity appears to approach 1,000,000 counts. EcoCounters can also be linked to a wireless network, and assuming connectivity to a wireless telephone network is available, data can be downloaded directly to a computer in an office. The TRAFx counters are very similar to TrailMaster counters but offer an option for a larger memory capacity. TRAFx counters can be set to collect 14,000 time stamped data pass-by events or to 
collect pass-by counts in 1-hour bins thereby increasing memory capacity to about 1.5 years of available memory.

The methods described in this paper were used to calibrate and analyze data from the EcoCounter and TRAFx counters. Results from these studies indicate that both of these devices behave similarly to the TrailMaster model in terms of counting errors. In other words, the EcoCounter and TRAFx counters systematically miscounted the number of people passing a study area but accurate and precise correction factors were derived using the protocols described in this paper. Furthermore, results indicate that EcoCounters miscounted arrivals and departures at different rates. Thus, unique correction factors were estimated for arriving and departing visitor counts to produce accurate estimates of visitor use. This finding is partially substantiated by Greene-Roesel et al. (2008) that found EcoCounters to undercount pass-by events by -9\% to $19 \%$ and suggested that correction factors be developed via direct observation to derive accurate estimates of pedestrian volumes.

\subsection{Implications}

These results show that automated visitor monitors can provide data to accurately estimate visitor use in parks and protected areas. All of the monitors used in this study were subject to counting errors and correction factors needed to be calculated from observed counts to calculate accurate estimates of visitor use. These results suggest that researchers and managers who choose to use automated monitors to estimate visitor use need to provide personnel to collect direct visitor counts. The necessity of this step cannot be underestimated because counts from automated monitors that are not calibrated cannot be considered a proxy for visitor use estimates. 
One hundred and thirty-five hours of direct observations were collected for the 6 monitors used in this study. Counts greater than zero were obtained during all observations because all of the study sites were high use areas. However, direct observations to calibrate automated monitors in low use areas may produce counts of zero during many observation periods. While a zero count provides relevant and useful data towards estimating correction factors, it is necessary to obtain a sufficient quantity of counts greater than zero in order to estimate the variability of a correction factor. Five hours of direct observations with counts greater than zero are suggested based on a post analysis of required sample size (as described in equation 1) of calibration data from Unit 1. Results from this post analysis showed that 5 hours of direct observations would produce an error of approximately 15\%. In addition, it is important to periodically monitor the condition and performance of automated visitor monitors in case of tampering by visitors or malfunction due to changing environmental conditions. Estimations of visitor use derived from automated visitor counters without estimating correction factors and corresponding standard errors should be considered suspect measures of visitation.

Managers who choose to collect visitor use data using automated visitor counters need to consider the costs of personnel training and field time as part of overall project expenditures. Training to set up counters and collect calibration data is relatively basic and about a half-day of training will cover the subject matter. The subsequent amount of time spent in the field by staff to collect calibration data is relative to the required level of precision by park management. As previously stated, we recommend at least 5 hours of direct observations, however, more observations are likely to produce more precise estimates of visitor use. Park managers should identify how these visitor use data will be used and the required level precision of estimates before counters are installed at a study site. 
The analytical process to derive correction factors and estimates of visitor use are not trivial and the amount of training required will depend on the quantitative analytical abilities of park staff. The calculations described in this paper can be accomplished using standard spreadsheet software such as Microsoft Excel but the use of analytical statistical software such as ' $\mathrm{R}$ ' can reduce the amount of time spent on data analysis. In addition, efficient management of the large amounts of data produced from automated visitor counters is imperative. Standard database programs such as Microsoft Access can be used to query and simplify large datasets. Thus, it may be useful for park managers to include time for information technologies (IT) staff as part of project costs to complete the more technical aspects of the analytical portions of this process.

\subsection{Limitations}

While automated monitors are a useful tool to estimate visitor use these devices are not without limitations. For example, automated visitor counters do not discriminate between different types of users. Additional sampling via direct observation or video recording is necessary to estimate the amounts and different types of users along a trail. In addition, visitor counts from automated visitor counters installed in wilderness areas where visitor use is very low may be skewed by wildlife passing through a monitor's detection range. In these cases, additional sampling may be required to understand typical visitor use patterns and their correlations to automated visitor counters.

\section{Conclusion}

Baseline data on visitor use is essential to the planning and management of national park units. Area administrators must be knowledgeable about the amount, types, locations, and behaviors of visitor use. Such information is fundamental to address a variety of visitor-use 
related issues, including visitor use capacity. Recently, automated visitor counters have become an accepted method for estimating visitor use in parks and protected areas. However, few studies provide thorough methodologies to correct raw monitor counts in order to calculate estimates of visitor use. This paper provides methodologies to obtain direct observation counts and calculate correction factors in order to estimate visitor use from automated visitor counters. Moreover, the automated visitor monitors used in this study performed consistently well at all study sites and during all time periods. The findings in this paper provide a methodological base for researchers and park managers interested in using automated visitor counters to estimate visitor use in parks and protected areas.

\section{List of References}

1. Arnberger, A., Haider, W., Brandenberg, C. (2005). Evaluating visitor-monitoring techniques: A comparison of counting and video observation data. Environmental Management, 36(2) 317327.

2. Bates, M., Wallace, G., \& Vaske, J. J. (2007). Estimating visitor use in Rocky Mountain National Park. (HDNRU Report No. 74). Report for Rocky Mountain National Park. Fort Collins: Colorado State University, Human Dimensions in Natural Resources Unit.

3. Chen, R.J.C., Bloomfield, P., \& Fu, J. (2003). An evaluation of alternative forecasting methods to recreation site use. Journal Leisure Res, 35, 441 - 454.

4. Crawley, M.J., (2005). Statistics an introduction using R. West Sussex, England: John Wiley \& Sons Ltd.

5. Dwyer, J.F., (1988). Predicting daily use of urban forest recreation sites. Landscape Urban Plan, 15, 127-138.

6. Gracia-Longares, M., (2005). Study of spatial patterns of visitors using mechanical counters, GPS, and GIS technology in the Slough Creek subregion of Yellowstone National Park. Unpublished Master's thesis, University of Montana, Bozeman, Montana, United States.

7. Greene-Roesel, R., Diogenes, M.C., Ragland, D. R., \& Lindau, L.A. (2008). Effectiveness of a commercially available automated pedestrian counting device in urban environments: 
comparison with manual counts. Berkeley: University of California Berkeley, Safe Transportation Research \& Education Center. Retrieved from: http://escholarship.org/uc/item/ 2n83w1q8

8. Lawson, S.R., (2006). Computer simulation as a tool for planning and management of visitor use in protected natural areas. Journal Sustain Tour, 14(6) 600-617.

9. Lawson, S.R., Kiser, B., Hockett, K., Reigner, N., Chamberlain, R., \& Choi, J., (2008). Visitor use computer simulation modeling to address transportation planning and user capacity management in Yosemite Valley, Yosemite National Park. Final Report for Yosemite National Park. Blacksburg, VA: Virginia Polytechnic Institute and State University, Forestry Department.

10. Lawson, S., Newman, P., Choi, J., Pettebone, D., \& Meldrum, B. (2009). Integrated transportation and user capacity research in Yosemite National Park, the numbers game. Transportation Research Board, 2119, 83-91.

11. Lindsey, P., \& Lindsey, G. (2004). Using pedestrian models to estimate urban trail traffic. The Journal of Regional Analysis \& Policy. 34(1), 51-68.

12. Lindsey, G. \& Nguyen, D.B.L. (2004). Use of greenway trails in Indiana. J Urban PlanningASCE, 130(4), 213-217.

13. Lindsey, G., Han, Y., Wilson, J., \& Yang, J. (2006). Neighborhood correlates of urban trail use. Journal of Physical Activity and Health, 3(S1), 139-157.

14. Lindsey, G., Wilson, J., Rubchinskaya, E., Yang, J., \& Han, Y., (2007). Estimating urban trail traffic: Methods for existing and proposed trails. Landscape Urban Plan, 81, 299-315.

15. Loomis, J.B. (2000). Counting on recreation use data: a call for long term monitoring. $J$ Leisure Res, 32(1), 93-96.

16. Lynch, J., Vogt, C., Cindrity, S., \& Nelson, C., (2002). Measuring and monitoring trail use: A nationwide survey of state and federal trail managers. Report for Michigan Department of Transportation (via the Pere Marquette Rail-Trail Research Project). Michigan State University, Department of Park, Recreation and Tourism Resources.

17. Manning, R.E., (2008). Parks and carrying capacity commons without tragedy. Corvalis, Oregon: Island Press.

18. Muhar, A., Arnberger, A., \& Brandenburg, C., (2002). Methods for Visitor Monitoring in Recreational and Protected Areas: An Overview. Conference proceedings from Monitoring and Management of Visitor Flows in Recreational and Protected Areas.

19. National Park Service, (2008). NPS stats, National Park Service public use statistics office. Retrieved December 1, 2008 from http://www.nature.nps.gov/stats/ 
20. Pettebone, D., Newman, P., Beaton, C., Stack, D., \& Gibson, A. (2008). Estimating visitor use in Yosemite National Park. Report for Yosemite National Park. Fort Collins: Colorado State University, Center for Protected Areas Management \& Training.

21. Scheaffer, R.L., Mendenhall, W., \& Ott, R.L., (2006). Elementary survey sampling (6th ed.). Belmont, CA: Duxbury.

22. Vaske, J. J. \& Donnelly, M. P., (2007). Estimating visitor use at Boulder Open Space and Mountain Parks: Summer 2004-2006 Comparisons. (HDNRU Report No. 79). Report for Boulder Open Space and Mountain Parks. Fort Collins: Colorado State University, Human Dimensions in Natural Resources Unit.

23. Vaske, J.J., Shelby, L.B, \& Donnelly, M.P., (2008). Estimating visitor use at Boulder Open Space and Mountain Parks. (HDNRU Report No. 80). Report for Boulder Open Space and Mountain Parks. Fort Collins: Colorado State University, Human Dimensions in Natural Resources Unit.

24. Watson, A.E., Cole, D.N., Turner, D.L., \& Reynolds, P.S., (2000). Wilderness recreation use estimation: A handbook of methods and systems. USFS General Technical Report RMRS-GTR-56., USDA, Forest Service, Rocky Mountain Research Station. Ogden, UT.

25. White, D. D. \& Aquino, J. F., (2008). Visitor perspectives toward transportation issues in Yosemite National Park. Report for Yosemite National Park. Phoenix, AZ: Arizona State University. 